\title{
Effect of uncertainty on the time course for selection of verbal name codes
}

\author{
M. E. BERRYHILL, K. KVERAGA, L. WEBB, and H. C. HUGHES \\ Dartmouth College, Hanover, New Hampshire
}

\begin{abstract}
Response selection takes time. Hick's law (Hick, 1952) predicts that the time course of response selection is a logarithmic function of the number of equally likely response alternatives. However, recent work has shown that oculomotor responses constitute noteworthy exceptions in that the latencies of saccades (Kveraga, Boucher, \& Hughes, 2002) and smooth pursuit movements (Berryhill, Kveraga, Boucher, \& Hughes, 2004) are completely independent of response uncertainty. This finding extends to the case in which the required response was known in advance (i.e., simple reaction times [RTs] were equivalent to choice RTs). In view of these results, we reevaluated reports that latencies to name visually presented digits (Experiment 1 ) and/or repeat aurally presented digits (Experiment 2) are similarly independent of the size of the response set. We found that naming latencies were equivalent for response set sizes from one to eight, but simple RTs (response set of one) were faster. Thus, the overlearned task of digit naming is indeed highly automatic but has not reached the level of automaticity characteristic of the oculomotor system.
\end{abstract}

Selecting the correct response from a set of alternative responses is typically a time-consuming cognitive operation. This fundamental aspect of human performance is reflected in the almost universal finding that simple reaction times (RTs) are faster than choice RTs. A generalization of the effects of response uncertainty on RTs is given by Hick's law, which states that the mean RT is proportional to the logarithm of the number of response alternatives (Hick, 1952; Schweickert, 1993). However, differences between choice RTs and simple RTs may not be entirely attributable to the time course of response selection; response selection is not the only component process required in the performance of choice RT tasks not required by simple RT tasks. Choice RTs also require stimulus identification before the correct response can be determined, whereas simple RTs only require detection of the imperative stimulus before a prespecified response can be initiated.

Kveraga, Boucher, and Hughes (2002) obtained a set of results for which it was likely that response selection processes were entirely responsible for increases in mean RT that accompanied increases in the number of equally likely response alternatives. In one experimental condition, Kveraga et al. measured saccadic RTs when the number of potential target locations varied from 1 to 8 in octave steps (one, two, four, and eight target locations). This location uncertainty produced a corresponding response uncertainty. The level of stimulus-response (S-R) uncertainty was constant within a block of trials but varied between blocks of trials. Circular annuli indicated the

Correspondence should be addressed to H. C. Hughes, Department of Psychological and Brain Sciences, Dartmouth College, 6207 Moore Hall, Hanover, NH 03755 (e-mail: hch@dartmouth.edu). possible target locations within any one block of trials. When one of these annuli was filled with a luminous disk, subjects were instructed to look at that location as quickly and accurately as possible. The results showed that saccadic RTs were completely unaffected by the number of potential target locations (see also Saslow, 1967). Not only did saccadic RTs violate Hick's law, they also violated the virtually universal finding that choice RTs are longer than simple RTs. Kveraga et al. also examined the effects of S-R uncertainty in a keypress task using identical stimulus conditions. In this second task, each target location was associated with a different key. S-R uncertainty varied across blocks of trials just as it had for the saccade task, and the potential target locations were again indicated by circular annuli. The results from the keypress task conformed to Hick's law and included a substantial difference between simple and choice RTs. The effects of S-R uncertainty observed with the keypress task cannot be attributed to processes associated with detection or identification of the targets, since these variables had no effect on saccades using an identical display. Therefore, both tasks required the same target detection and identification components, and both required a different response be associated with each individual stimulus. The tasks differed only in the modality of the response. Kveraga et al. concluded that, in this instance, the increases in manual choice RTs can be attributed to the time taken to select the appropriate motor response.

Kveraga et al. (2002) suggested that saccadic responses are automatically accessed by the targets that evoke them. They hypothesized that automatic selection of saccadic amplitude and direction is made possible by the manner in which visual inputs are converted into motor commands in the oculomotor system. More specifically, they hypothesized that automatic selection of saccadic trajectories is 
attributable to the anatomical organization of the superior colliculus (SC) - a midbrain structure that is prominently involved in the production of visually guided saccades. The SC receives extensive visual inputs originating both from the retina and from the visual cortex. Like most visually responsive neurons, those in the SC possess spatially circumscribed receptive fields. Neighboring SC neurons have adjacent receptive field locations such that, in the aggregate, they form an orderly map of visual space (i.e., a retinotopic map). Like many neural structures, the SC is composed of several anatomically distinct cellular layers (Schiller \& Stryker, 1972; Wurtz \& Mohler, 1976). The visually responsive cells occupy the more superficial of these layers (for reviews, see Sparks, 1988, 1999, and Stein, Wallace, \& Meredith, 1995). The SC also contains premotor neurons that project to saccade-generating circuits in the brainstem and discharge in advance of saccadic eye movements (Basso \& Wurtz, 1997, 1998; Glimcher \& Sparks, 1992). These premotor neurons occupy the deeper layers of the SC. The presaccadic activity of these premotor cells is selective in that each cell's activity is dependent on the specific amplitude and direction of the movement. Each premotor neuron is tuned to a specific trajectory that is termed the cell's motor field. Like their visual counterparts, these motor fields are arranged in an orderly map within the deeper cellular layers of the SC (Gordon, 1973; Wallace, Wilkinson, \& Stein, 1996). An important feature of the organization of the SC is that the more superficial visual map and underlying motor map are in spatial register - the movement fields of the premotor cells are aligned with the visual receptive fields of cells that lie above them. This alignment between the visuotopic map and the motor map appears specifically designed to fixate eccentric visual targets. Interestingly, Berryhill et al. (2004) found that the latencies of smooth pursuit eye movements are also independent of S-R uncertainty (in this case, the possible directions of target motion). Recent findings suggesting that saccades and slow pursuit share a great deal more premotor circuitry than has previously been assumed may account for the automatic selection in both the saccadic and the pursuit control systems (Basso, Krauzlis, \& Wurtz, 2000; Krauzlis, Basso, \& Wurtz, 1997). If the coregistration of sensory and motor maps is responsible for immunity from Hick's law, then an absence of such tight sensorimotor coupling might require control operations that result in the time costs that accompany choice RTs. Since there is no indication of analogous sensorimotor connections controlling effectors, such as the digits of the hand or the arms, such high levels of automatic response selection should be very uncommon.

Yet eye movements are not the only response system reported to be immune to S-R uncertainty. Vocal RTs to name digits presented visually (e.g., Alluisi, Muller, \& Fitts, 1957; Regan, 1981; Theios, 1975) or aurally (Davis, Moray, \& Treisman, 1961) also reportedly violate Hick's law. We were interested in these reports because it appears unlikely that the anatomical place-coding explanation offered by Kveraga et al. (2002) for saccades is applicable to these naming tasks. Automatized access of name codes is usually attributed to overlearning of specific S-R connections that eventually produce a state of very high S-R compatibility (e.g., Davis et al., 1961; Fitts, 1964; Mowbray, 1960; Mowbray \& Rhoades, 1959; Regan, 1981; Theios, 1975). Although it had been widely believed that naming latencies are insensitive to $\mathrm{S}-\mathrm{R}$ uncertainty, there remain indications that the issue may not be fully resolved.

Stanovich and Pachella (1976) suggested that the data reported by Theios (1975) may have been contaminated by a trade-off of accuracy for speed for the high S-R uncertainty condition. In addition, the RTs reported by Theios seem rather slow $(>450 \mathrm{msec})$, raising the possibility that subjects may not have been optimizing their performance. Finally, Fitts and Switzer (1962) reported that digit-naming latencies actually followed Hick's law under what they considered conditions of high familiarity (two-, four-, and eight-choice RTs for naming the digits 1 and 2) but not under conditions of lower familiarity (two-, four- and eight-choice RTs for naming the digits 4 and 7). This is not the result that would be expected if automaticity of response selection was related to familiarity with the stimuli. However, the uncertainty effect in the "highfamiliarity" condition was quite modest (about $20 \mathrm{msec}$ ), and a statistically reliable effect has not been consistently observed (e.g., Regan, 1981; Theios, 1975). These reports make it clear that a highly overlearned task, such as digit naming, is largely unaffected by the size of the response set, although several factors influencing the subject's cognitive set can have modest effects (Fitts \& Switzer, 1962; Regan, 1981).

The most surprising aspect of the results reported by Kveraga et al. (2002) and Berryhill et al. (2004) is the observation that choice RTs were equivalent to simple RTs. This is almost never observed: two-choice RTs are typically 100-150 msec slower than simple RTs (e.g., Luce, 1986). The generally accepted explanation attributes this difference to the additional time it takes to select the correct response. For simple RTs, the correct response is known in advance and can therefore be programmed in advance, held in a high state of readiness, and triggered upon detection of the imperative stimulus. For choice RTs, the required responses are known in advance, and the subject may attempt to maintain the responses at a certain state of readiness, but this is assumed to be much less than is possible for simple RTs. In addition, choice RTs require selection of the correct response given a specific imperative stimulus. Thus, the results of Kveraga et al. and Berryhill et al. imply that the time taken to select among different oculomotor responses (either saccadic directions or smooth pursuit directions) is so negligible that it could not be detected. In order to determine whether naming latencies have achieved a comparable degree of automaticity, it is essential to compare simple and choice RTs. In previous experiments examining naming latencies, the simple RT condition was not included.

In the present experiments, we therefore reexamined RTs to vocalize the names of visually presented digits 
(Experiment 1) or to echo the names of aurally presented digits (Experiment 2) as a function of the size of the response set including a simple RT condition.

\section{EXPERIMENT 1 \\ Effects of Stimulus-Response Uncertainty on Naming Latencies for Visually Presented Digits}

This experiment is a replication and extension of earlier reports that digit-naming latencies are independent of the number of potential targets (digits to be presented). However, it differs from those earlier reports in two important respects. First, we included a condition in which the stimulus set size was one (stimulus and response known in advance). Second, subjects were provided with monetary performance incentives in an attempt to ensure that they were optimizing both the speed and the accuracy of their responses.

\section{Method}

Subjects. Six undergraduates (4 female, 2 male) participated in this experiment. They ranged in age from 18 to 21 years. All reported having normal visual acuity, and all were native speakers of English. They were compensated for their time, obtaining credit for experimental participation in an introductory psychology course at Dartmouth College or earning $\$ 4$ for each 0.5 -h experimental session (their choice). The experimental protocol was approved by the Dartmouth College Committee for the Protection of Human Subjects, and each subject signed an informed consent document.

Stimuli. The stimuli consisted of a set of integers ranging from 1 to 8 . The digits were white Arabic numerals presented against a black background on a 19-in. CRT display. They had a luminance of $27 \mathrm{~cd} / \mathrm{m}^{2}$ and subtended $1.8^{\circ}$ at a viewing distance of $57 \mathrm{~cm}$. We controlled the viewing distance by asking the subjects to lean against a forehead rest (we avoided the use of a chinrest because we did not want to interfere with the subjects' ability to say the digit names). The stimuli were presented for $100 \mathrm{msec}$ ( 6 raster scans at $16.67 \mathrm{msec} / \mathrm{scan}$ ). Stimulus presentation and timing were controlled by a PC running DOS. The presentation software included a subroutine that yoked stimulus presentation to the vertical refresh of the video monitor. This allowed us to precisely control the timing and duration of all stimuli. This stimulus presentation routine also set a bit in the machine's parallel port synchronously with the vertical retrace signal for the first video raster frame that contained the stimuli. This signal was used to determine the RTs (see the Response Recording section, below).

Response recording. Verbal responses were recorded using an audio microphone. The amplified output of the microphone provided input to a voltage comparator that produced a digital signal that marked the onset of the verbal responses (i.e., a voice-activated switch). The audio output of the microphone was also monitored by an experimenter located in a separate room. The experimenter listened to the responses and manually scored them for accuracy. In addition to the computer that controlled the sequence of stimuli, a second computer was used to digitize signals from the parallel port of the stimulus presentation machine as well as the output of the voice-controlled switch at the sampling rate of $1000 \mathrm{~Hz}$. This data acquisition computer was used to compute the RTs and saved the data for later analyses. RTs were accurate to the nearest millisecond.

Procedure. Each trial began with the presentation of a warning signal (a fixation cross presented at the center of the viewing screen), which remained for a random fixation interval of 500-1,500 msec before the digit appeared. Unpredictable onset time was imposed in order to prevent anticipatory responses in the one-target condition. The digits were randomly selected from a set size of one, two, four, or eight possible digits. The stimulus set size varied across blocks of trials pseudorandomly (random selection from a set size of one, two, four, or eight without replacement). There were 16 trials in a block when the set size was one, and there were two of these 16-trial blocks in each experimental session. In one 16-trial block, the stimulus presented was always the numeral 1 . In the other block of 16 trials, the numeral 2 was presented on every trial. There were 32 trials in each block when the set size was two. In this condition, the stimulus was either a 1 or a 2 , and they were each presented with a probability of .5. There were 64 trials for the four-item set. The stimuli in this case were drawn with equal probability from the set $\{1,2,3,4\}$. Finally, the eight-item set consisted of a block of 128 trials in which the stimuli were selected from the set $\{1,2,3,4,5,6,7,8\}$, each with a probability of .125. In all cases, the task was simply to name the stimulus as quickly and accurately as possible. At the beginning of each block, the subjects were shown the set of digits from which the stimuli would be drawn during that particular block (e.g., 1, or 1,2 , etc.). The subjects participated in two practice sessions and four experimental sessions. Each session consisted of two blocks of trials using an S-R set size of one (1 and 2) and three blocks using each of the remaining S-R set sizes of two, four, and eight alternative stimuli. At the beginning of the first session, the subjects were informed about the general aspects of the task and were instructed to respond as quickly and accurately as possible. They were told that any block in which their performance fell below $95 \%$ correct would have to be rerun (this was never necessary). At the beginning of the second practice session, the subjects were encouraged to try to improve their levels of performance beyond what they produced during the first session. The average RT for this second practice session served as a benchmark for evaluating all subsequent experimental sessions. After this second practice session was completed, the subjects were told that they could earn a financial performance payoff of $\$ 1$ for each block of trials in which their average RT fell below their previously established performance criterion, so long as their accuracy remained above $95 \%$ correct. At the end of each block, the subjects were given performance feedback as to whether or not they had beaten their target performance level; at the end of each session, they were given their performance rewards for that session.

\section{Results}

Response accuracy. The average accuracy for all subjects exceeded $99.5 \%$, and no individual subject's accuracy ever fell below $98 \%$ correct in any block of trials. Since the subjects' responses were essentially error free, we did not analyze the response accuracy data further.

Response times. RTs less than $150 \mathrm{msec}$ or greater than $800 \mathrm{msec}$ were excluded from further analysis. Application of these cutoffs removed less than $1.0 \%$ of the trials from the data set. We confined our analysis of the latency data to the two responses that were common to all four S-R uncertainty conditions (the naming latencies for the numerals 1 and 2). The mean RTs are plotted as a function of S-R set size in Figure 1, which indicates that S-R uncertainty exerted a substantial effect on vocal RTs. This was confirmed by a three-factor repeated measures ANOVA. The experimental factors were the four levels of S-R uncertainty (one, two, four, or eight potential digits), four levels of practice (Sessions 1-4), and two levels of stimulus identity (1 or 2). The main effects of S-R uncertainty $[F(3,15)=64.54, p<.01]$, practice $[F(3,15)=$ $25.28, p<.01]$, and stimulus identity $[F(1,5)=7.24, p<$ 


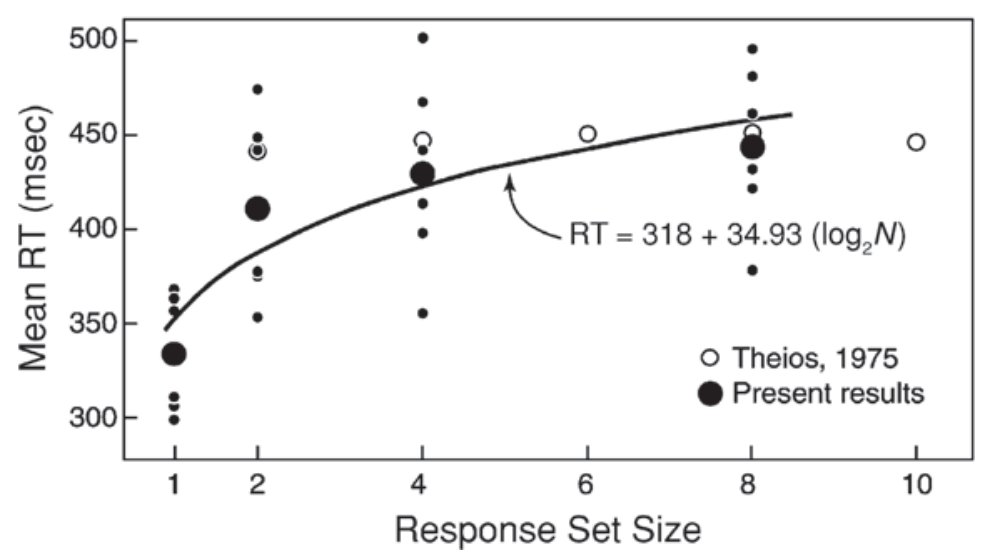

Figure 1. Mean reaction time (RT) as a function of response set for 6 observers in Experiment 1. Data from Theios (1975) are included for comparison purposes.

$.05]$ were all significant. None of the interactions were statistically significant. Post hoc comparisons indicated that simple RTs (S-R set size of one) were significantly faster than all other set sizes, and that the RTs for S-R set size of two was significantly faster than RTs for S-R set size of eight.

In order to evaluate the nature of the practice effect, the data from each subject in each experimental session was fit to a function having the form RT $=b+a\left(\log _{2} N\right)$, where $N$ is the number of response alternatives. The groupaveraged parameters for the best-fitting functions for each experimental session are provided in Table 1 .

The majority of the improvement clearly occurred between the first and second experimental sessions, and the general effect was to produce a uniform decrease in RTs for all S-R uncertainty conditions (the effect was confined to the intercept of the best-fitting functions and did not systematically influence the slopes). Despite the apparent trends in Table 1, two two-way ANOVAs (response ["1" vs. " 2 "] and sessions [1-4]) performed on the slopes and intercepts of these best-fitting parameters revealed no significant main effects and no interactions (all $p \mathrm{~s}>$.299).

\section{Discussion}

In contrast to previous results (e.g., Alluisi et al., 1957; Davis et al., 1961; Morin, Konick, \& McPherson, 1965; Regan, 1981; Theios, 1975), the present results indicate that S-R uncertainty lengthens the naming latencies of

Table 1

Summary of Practice Effects on Naming Visually Presented Digits in Experiment 1

\begin{tabular}{ccc}
\hline Session & Slope & Intercept \\
\hline 1 & 31.0 & 397.6 \\
2 & 34.7 & 356.2 \\
3 & 40.8 & 355.0 \\
4 & 33.6 & 349.4 \\
\hline
\end{tabular}

visually presented digits. There was a substantial difference between simple and two-choice RTs (77 msec), and there was an additional 33-msec effect between two and eight response alternatives. However, it must be acknowledged that the major effect on RT occurs at the transition between simple and choice RTs (one and two responses alternatives). Beyond that notable exception, the present results are largely consistent with the earlier reports.

The essential difference between choice and simple RTs is that the response is known in advance of the imperative stimulus for simple RTs but must be selected on the basis of identity of the imperative stimulus for choice RTs. Since the simple RT response is known in advance, it can be prepared in advance (preprogrammed) and can then simply be triggered on detection of the imperative stimulus. It seems clear that most of the difference between choice and simple RTs is due to time-consuming cognitive operations of stimulus identification and response selection. Figure 2 is a schematic illustration of this idea. Although the figure depicts serial processing, it need not imply strictly serial stages in which processing of later stages cannot begin until earlier processes have been completed. That is, cascaded serial stages are equally compatible with this general concept insofar as they also predict that simple RTs will be faster than choice RTs.

In its simplest form, the serial model of choice RT illustrated in Figure 2 predicts that the relationship between RT and response uncertainty should be a step function. A step increase occurs at the transition from simple to choice RTs because choice RTs require cognitive operations (in Figure 2, they are stimulus identification, response selection, and motor programming) that are not required on a trial-by-trial basis in simple RT tasks. Note that there is no a priori reason why response uncertainty per se should have any effect beyond two alternative choices because the same cognitive operations (stimulus identification, response selection, and motor programming) are required irrespective of the number of potential responses. If the 

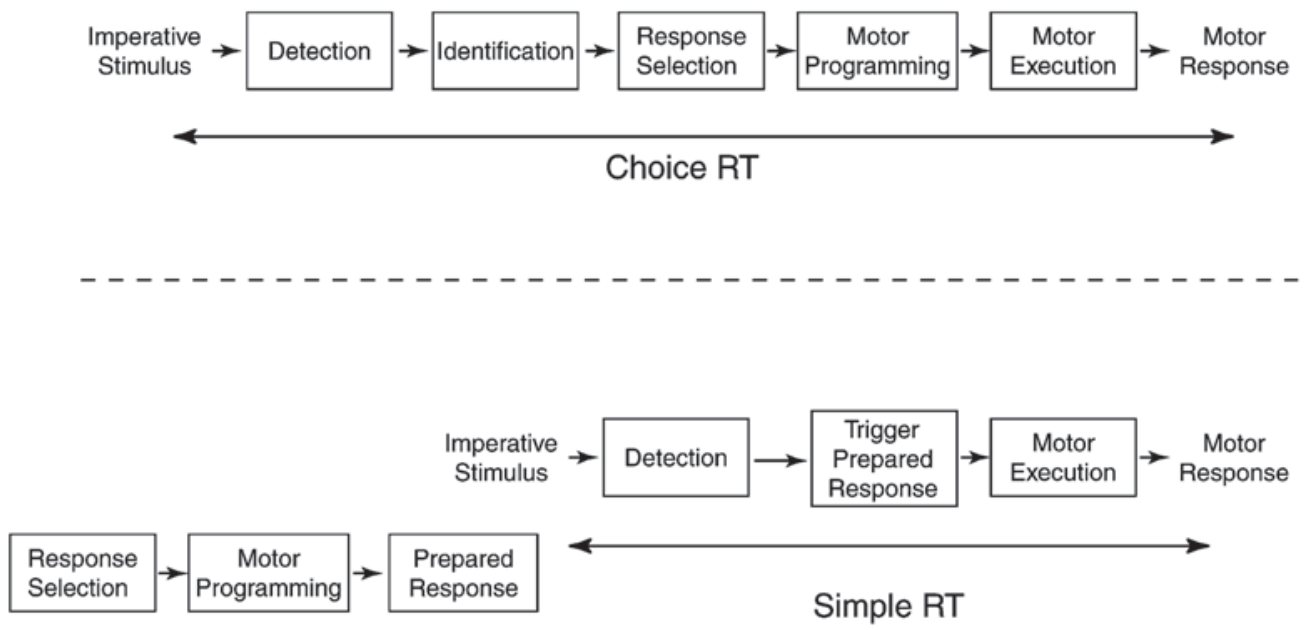

Figure 2. Serial stage models of the cognitive operations required by simple and choice reaction time (RT) tasks.

process of response selection involves some sort of serial search through the response set (e.g., Theios, 1975, for a description of such a model) or the distribution of a limited-capacity central resource across the set of potential S-R connections, then RTs should increase monotonically with the size of the response set, as predicted by Hick's law. However, it is commonly assumed that the S-R connections for highly overlearned tasks such as digit naming have become so ingrained through extensive practice that inputs automatically access the appropriate response codes, bypassing the time-consuming control processes that would otherwise be needed to access the correct motor response (e.g., Regan, 1981; Theios, 1975). Under these circumstances, any effect that the size of the response might have on the time course of response selection might be negligible relative to the means and variances of other component operations, making the effect on the overall RTs difficult to detect. The benefit of having a preprogrammed response might still be apparent, however. Thus, for highly skilled tasks, many conceivable implementations of Figure 2 could predict a step-function relationship between mean RT and S-R uncertainty-they only need postulate that the time course of response selection is effectively independent of the size of the response set. Figure 3 illustrates that the present data are reasonably well fit by a step function comprising two segments with a slope equal to zero. A discrete change in RT occurs between one and two response alternatives, but RTs do not continue to rise with more than two alternatives. This step function accounts for $48 \%$ of the variance in the individual subject means illustrated in Figure 1. In comparison, the logarithmic fit accounts for $44 \%$ of the variance; so, by this criterion, the present data are not sufficient to reject either Hick's law or a step-function fit.

Of course, to conclude that a step function fits the data as well as logarithmic function ignores the statistically significant difference between the two- and eight-alternative conditions, but that particular effect is small in the pres-

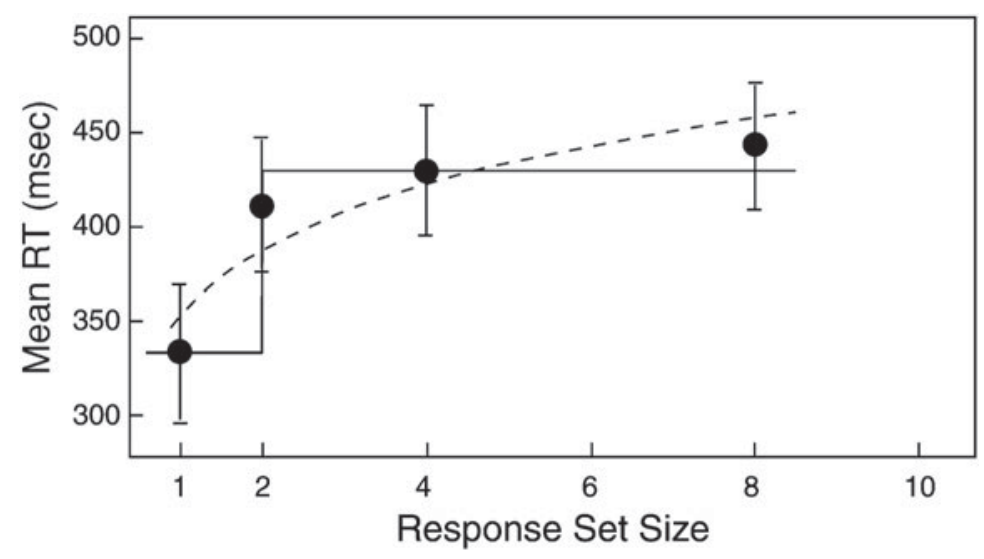

Figure 3. A comparison between a logarithmic and step-function fit of the data in Experiment 1. Error bars represent the standard error of the mean. 
ent data and has not appeared in many previous experiments (e.g., Regan, 1981; Theios, 1975). For the moment, it seems we must remain agnostic on this point.

\section{Comparison Between Digit Naming Latencies and Latencies of Saccadic Eye Movements}

One of the motivations behind the present reexamination of digit-naming latencies derives from Kveraga et al.'s (2002) finding that saccadic eye movements are immune to the effects of S-R uncertainty (extending from eight-choice RTs to simple RTs). These authors attempted to relate the automaticity of saccadic eye movements to known properties of the superior colliculus. They suggested that fixed connections between visually responsive neurons that are driven by the saccade target and premotor command neurons that drive the eyes to the location of the target imply that the selection of saccadic parameters of direction and amplitude is anatomically coded in such a way that visual stimuli automatically access the appropriate motor program.

An alternative explanation attributes this immunity to extensive practice (e.g., Regan, 1981; Seibel, 1963). It may be possible that any highly practiced skill can achieve the level of automaticity reported for saccades (Kveraga et al., 2002) and smooth pursuit eye movements (Berryhill et al., 2004). Earlier work vividly demonstrates the remarkable effects that extensive practice can exert over the relationship between processing time and response uncertainty (Seibel, 1963). However, it appears that despite years of practice, our selection of verbal name codes cannot achieve the phenomenal degree of automaticity seen for saccades and smooth pursuit eye movements.

Although practice effects were evident in the present data, the effect of practice was to produce a uniform decrease in mean RTs for all response uncertainty conditions (including simple RTs). The opposite effect - a uniform increase in RT across response set sizes (two, four, and eight) — was reported by Regan (1981), who investigated naming latencies when Armenian letters were mixed with English letters. This mixed condition elevated RTs for naming English letters relative to a condition in which only English letters were presented. Thus, both context and practice within the experimental setting can influence naming latencies, but the effects tend to be equivalent across all response set sizes and, therefore, do not alter the basic conclusions regarding the effect of response uncertainty.

We conclude that accessing verbal name codes is indeed highly automatized. However, the process of selecting and retrieving lexical motor programs apparently is not as automatized as the process of selecting and programming eye movements. Thus, unlike saccade or smooth pursuit performance, digit naming performance benefits when motor programs can be activated in advance of the imperative stimulus, so simple RTs are faster than choice RTs. Figure 4 provides a direct comparison of the present findings with the saccadic results reported by Kveraga et al. (2002).

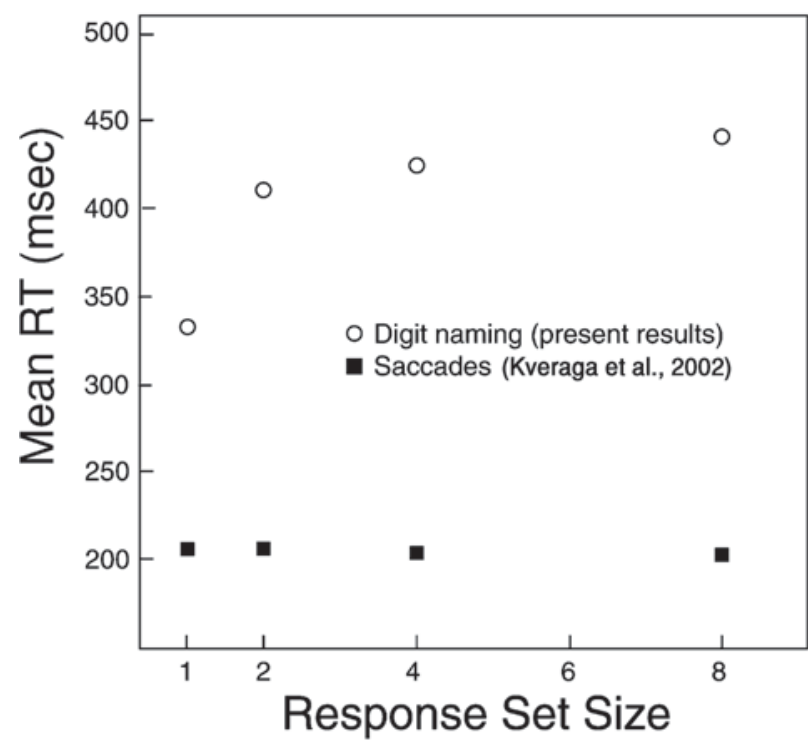

Figure 4. Comparison between group mean reaction times (RTs) for naming visually presented digits (Experiment 1) and the results from Kveraga et al. (2002).

\section{EXPERIMENT 2 \\ Effects of Stimulus-Response Uncertainty on Naming Latencies for Auditory Digits}

Experiment 2 was the auditory counterpart of Experiment 1 . The primary goal of this second experiment was the same as that of Experiment 1, to extend the analysis of the effects of S-R uncertainty in echoing auditory digits to the case of simple RTs. We hypothesized that the results might differ for auditory digit naming because of a closer relationship between auditory processing and premotor phonological processing. We reasoned that this might occur because of the natural feedback loop between vocalizations and the auditory system (McGuire, Silbersweig, \& Frith, 1996; Oomen \& Postma, 2001; Shuster \& Durrant, 2003) or because of the "privileged loop," whereby echoing spoken words is thought to bypass certain aspects of speech production (McLeod \& Posner, 1984). One hypothesis is that this constant and consistent feedback from individuals' own vocalizations may lead to a state in which auditory inputs automatically activate the corresponding phonological code, and this state may have a unique level of access to the speech apparatus. If so, response uncertainty may have less influence on vocal RTs if the stimuli are presented in the auditory modality. In Experiment 2, we examined that possibility.

\section{Method}

Subjects. Six undergraduates ( 3 female, 3 male) participated in this experiment. They ranged in age from 18 to 21 years. All reported having normal hearing, and all were native speakers of English. They were compensated for their time, obtaining credit for experimental participation in an introductory psychology course at Dartmouth College or earning $\$ 4$ for each 0.5 -h experimental session (their choice). The experimental protocol was approved by the Dartmouth 


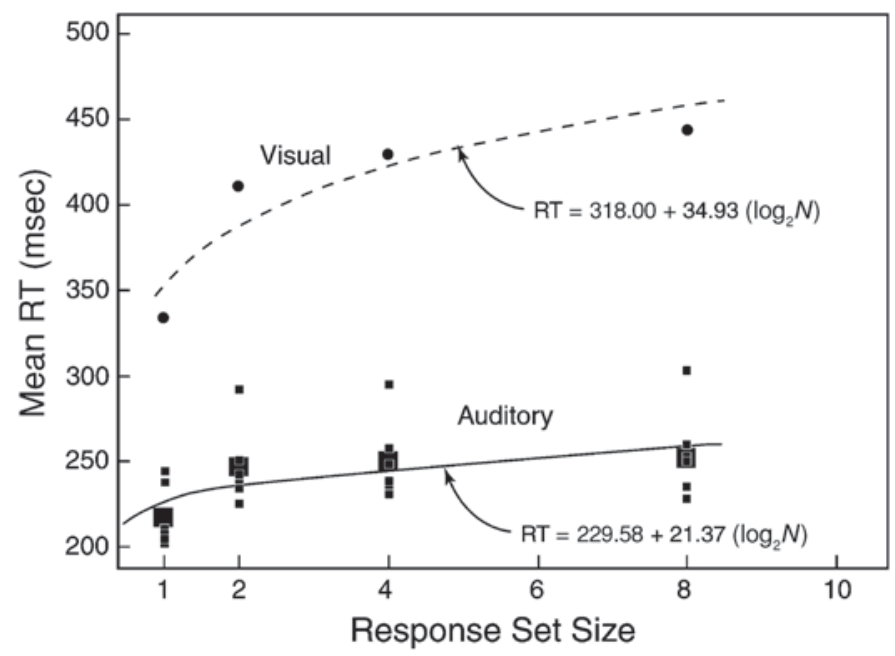

Figure 5. Naming reaction times (RTs) for acoustically presented digits as a function of $\mathrm{S}-\mathrm{R}$ uncertainty. Large squares indicate group means, and small squares indicate individual subject means. Summary data from Experiment 1 are included for comparison.

College Committee for the Protection of Human Subjects, and each subject signed an informed consent document.

Stimuli. The stimuli consisted of a set of spoken digit names ranging from 1 to 8 . The auditory stimuli were digitized recordings of a female voice speaking the name of each numeral and were presented using a standard PC sound card and computer speaker system.

Response recording. Verbal responses were recorded using the same setup as that in Experiment 1. As in Experiment 1, the experimenter listened to the responses and manually scored them for accuracy. Both stimulus presentation and data acquisition were accomplished using a commercial data acquisition software system (LabVIEW, National Instruments Corp.). RTs were accurate to the nearest millisecond.

Procedure. With the exception of the modality of the stimuli, the procedure was identical to that used in Experiment 1. Each trial began with the presentation of a warning signal, which consisted of a fixation cross presented at the center of the viewing screen. The fixation cross remained visible throughout the duration of the foreperiod, which was selected from a uniform distribution covering the interval between 500 and 1,500 msec. Auditory digit names were randomly selected from a set size of one, two, four, or eight possible digits. The stimulus set size varied across blocks of trials pseudorandomly, as in Experiment 1. The task was simply to echo the name of the stimulus as quickly and accurately as possible. At the beginning of each block, the set size was indicated to the subjects, who were shown the set of digits from which the stimuli would be drawn during that particular block (e.g., 1, or 1, 2, etc.).

\section{Results}

Response accuracy. The average accuracy rate for all 6 subjects in four uncertainty conditions was $99.8 \%$. Accuracy never fell below $99 \%$ for any subject in any uncertainty condition.

Reaction times. RTs less than $150 \mathrm{msec}$ or greater than $800 \mathrm{msec}$ were excluded from further analysis. This removed less than $1.0 \%$ of the trials from the data set. We again confined our analysis of the latency data to the two responses that were common to all four $\mathrm{S}-\mathrm{R}$ uncertainty conditions: the naming latencies for aurally presented 1 and 2. The mean RTs are plotted as a function of S-R set size in Figure 5, which indicates that (1) naming RTs were substantially faster for auditory stimuli than for visual stimuli (data from Experiment 1 are included for comparison) and (2) S-R uncertainty did indeed affect vocal RTs. The effect of S-R uncertainty was confirmed by a threefactor repeated measures ANOVA. The experimental factors were the four levels of S-R uncertainty (response sets of one, two, four, or eight), four levels of practice (Sessions $1-4)$ and two levels of stimulus identity ( 1 or 2$)$. The main effects of S-R uncertainty $[F(1,24)=23.63, p<.01]$ and practice $[F(2,6)=8.73, p<.01]$ were both significant, but the main effect of stimulus identity was not $[F(1,5)=$ $2.96, p=.15]$. None of the interactions were significant. As in Experiment 1, we evaluated the practice effect further by fitting the data from each experimental session to the function RT $=b+a\left(\log _{2} N\right)$, where $N$ is the number of response alternatives. The best-fitting slopes and intercepts are provided in Table 2. As in Experiment 1, the effect of practice was to reduce the overall baseline level of performance without having much impact on the relative effects of response entropy. As above, additional two-factor ANOVAs examined the effects of response type (1 vs. 2)

Table 2 Summary of Practice Effects on Naming Visually Presented Digits in Experiment 2

\begin{tabular}{ccc}
\hline Session & Slope & Intercept \\
\hline 1 & 26.5 & 241.4 \\
2 & 21.1 & 233.6 \\
3 & 20.3 & 219.5 \\
4 & 19.3 & 220.5 \\
\hline
\end{tabular}




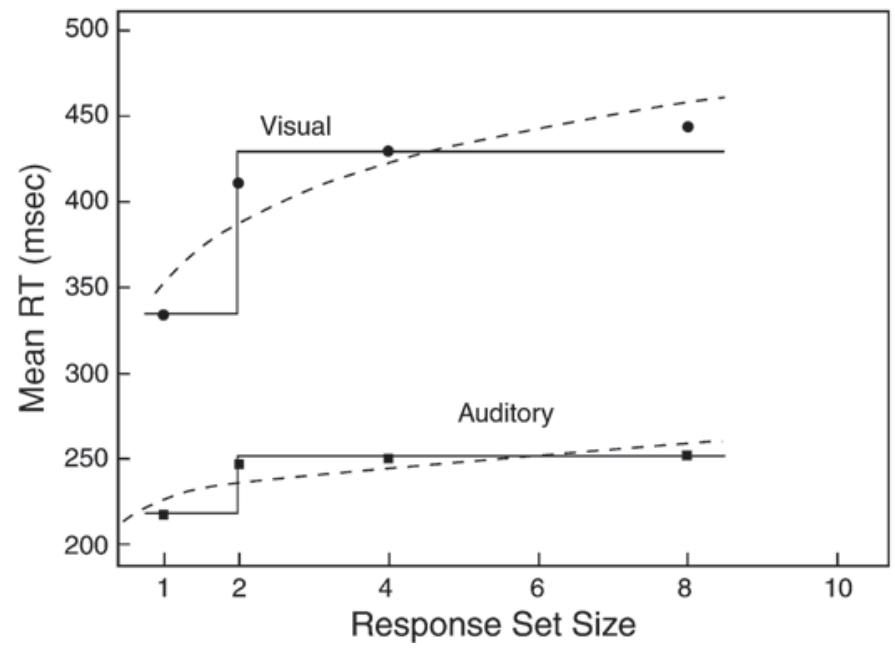

Figure 6. Comparison of log and step-function fits. Both the auditory data and the visual data are similarly fit by both a step function and a $\log$ fit function.

and experimental session on the slope and intercept of the best fitting functions and found no significant main effects or interactions.

Post hoc comparisons indicated that simple RTs (S-R set size $=1$ ) were significantly faster than all other set sizes, but all other levels of S-R uncertainty were statistically indistinguishable. The best fitting logarithmic function is illustrated in Figure 5. That function accounts for $22 \%$ of the variance in the individual subject means. Figure 6 compares the log fit and the step-function fit. It is clear that once again the data do not permit selection of one model function over the other $\left(\log r^{2}=.22\right.$; stepfunction $r^{2}=.29$ ).

\section{GENERAL DISCUSSION}

The results of Experiment 2 were very similar to those of Experiment 1. The main effect of response uncertainty occurred in the transition from simple to choice RTs. Although there was some suggestion that S-R uncertainty had a modest effect on performance for set sizes greater than 2 in Experiment 1, there was no indication of that in Experiment 2.

The experiments reported here were designed to determine whether previous reports that digit naming violates Hick's law extend to the case of simple RTs as it does for saccades (Kveraga et al., 2002) and smooth pursuit eye movements (Berryhill et al., 2004). The present results replicate previous work indicating that digit naming latencies are essentially independent of response sets that span the range between two and eight (or more) alternatives. This is true of naming visually presented digits (e.g., Alluisi, Strain, \& Thurmond, 1964; Theios, 1975), as well as "echoing" digits presented acoustically (Davis et al., 1961). In Experiment 1, we found a significant difference between the two- and eight-alternative response condi- tions, but the absolute magnitude of the effect was small, and it was not replicated using auditory stimuli in Experiment 2. Logarithmic fits of the data were no better than step-function fits, suggesting that the effects of response uncertainty on naming latencies are extremely modest beyond two response alternatives. However, this relative immunity to higher order S-R uncertainty clearly does not extend to response sets of one, and, in this important respect, the process of reading visually presented digits aloud or echoing aurally presented digit names is less automatized than the oculomotor tasks studied by Kveraga et al. and Berryhill et al. The oculomotor system may be unique in that there is no performance benefit from the ability to preprogram a motor response; that is, there is no difference between choice and simple RTs.

\section{REFERENCES}

Alluisi, E. A., Muller, P. F., JR., \& Fitts, P. M. (1957). An information analysis of verbal and motor responses in a forced-paced serial task. Journal of Experimental Psychology, 53, 153-158.

Alluisi, E. A., Strain, G. S., \& Thurmond, J. B. (1964). Stimulusresponse compatibility and the rate of gain of information. Psychonomic Science, 1, 111-112.

Basso, M. A., Krauzlis, R. J., \& Wurtz, R. H. (2000). Activation and inactivation of rostral superior colliculus neurons during smoothpursuit eye movements in monkeys. Journal of Neurophysiology, 84, 892-908.

Basso, M. A., \& Wurtz, R. H. (1997). Modulation of neuronal activity by target uncertainty. Nature, 389, 66-69.

Basso, M. A., \& Wurtz, R. H. (1998). Modulation of neuronal activity in superior colliculus by changes in target probability. Journal of Neuroscience, 18, 7519-7534.

Berryhill, M. E., Kveraga, K., Boucher, L., \& Hughes, H. C. (2004). Smooth pursuit under stimulus-response uncertainty. Cognitive Brain Research, 19, 100-102.

Davis, R., Moray, N., \& Treisman, A. (1961). Imitative responses and the rate of gain of information. Quarterly Journal of Experimental Psychology, 13, 78-89.

FitTs, P. M. (1964). Perceptual-motor skill learning. In A. W. Melton 
(Ed.), Categories of human learning (pp. 243-285). New York: Academic Press.

FitTs, P. M., \& Switzer, G. (1962). Cognitive aspects of information processing: I. The familiarity of S-R sets and subsets. Journal of Experimental Psychology, 63, 321-329.

GLimcher, P., \& Sparks, D. L. (1992). Movement selection in advance of action in the superior colliculus. Nature, 355, 542-545.

Gordon, B. (1973). Receptive fields in deep layers of cat superior colliculus. Journal of Neurophysiology, 36, 157-178.

Hick, W. E. (1952). On the rate of gain of information. Quarterly Journal of Experimental Psychology, 4, 11-26.

Krauzlis, R. J., Basso, M. A., \& Wurtz, R. H. (1997). The rostral superior colliculus influences pursuit eye movements in the primate as well as fixation and saccades. Investigative Ophthalmology \& Visual Science, 8 (Suppl.), 932.

Kveraga, K., Boucher, L., \& Hughes, H. C. (2002). Saccades operate in violation of Hick's law. Experimental Brain Research, 146, 307-314.

LUCE, R. D. (1986). Response times: Their role in inferring elementary mental organization. New York: Oxford University Press.

McGuire, P. K., Silbersweig, D. A., \& Frith, C. D. (1996). Functional neuroanatomy of verbal self-monitoring. Brain, 119, 907-917.

McLeod, P., \& Posner, M. I. (1984). Privileged loops from percept to act. In H. Bouma \& D. Bouwhuis (Eds.), Attention and performance $X$ (pp. 55-66). Hillsdale, NJ: Erlbaum.

Morin, R. E., Konick, A., \& McPherson, S. (1965). Information and reaction time for naming responses. Journal of Experimental Psychology, 70, 309-314.

Mowbray, G. H. (1960). Choice reaction times for skilled responses. Quarterly Journal of Experimental Psychology, 7, 193-202.

Mowbray, G. H., \& Rhoades, M. V. (1959). On the reduction of choice reaction times with practice. Quarterly Journal of Experimental Psychology, 6, 16-23.

Oomen, C. C. E., \& Postma, A. (2001). Effects of time pressure on mechanisms of speech production and self-monitoring. Journal of Psycholinguistic Research, 30, 163-184.

REGAN, J. E. (1981). Automaticity and learning: Effects of familiarity on naming letters. Journal of Experimental Psychology: Human Perception \& Performance, 7, 180-195.

SASLOW, M. G. (1967). Latency for saccadic eye movement. Journal of the Optical Society of America, 57, 1030-1033.
Schiller, P. H., \& Stryker, M. P. (1972). Single-unit recording and stimulation in superior colliculus of the alert rhesus monkey. Journal of Neurophysiology, 35, 915-924.

SCHWEICKERT, R. (1993). Information, time, and the structure of mental events: A twenty-five-year review. In D. E. Meyer \& S. Kornblum (Eds.), Attention and performance XIV: Synergies in experimental psychology, artificial intelligence, and cognitive neuroscience (pp. 535-566). Cambridge, MA: MIT Press.

SEIBEL, R. (1963). Discrimination reaction time for a 1,023-alternative task. Journal of Experimental Psychology, 66, 215-226.

Shuster, L. I., \& DuRRANT, J. D. (2003). Toward a better understanding of the perception of self-produced speech. Journal of Communication Disorders, 36, 1-11.

SPARKS, D. L. (1988). Neural cartography: Sensory and motor maps in the superior colliculus. Brain Behavior \& Evolution, 31, 49-56.

SPARKS, D. L. (1999). Conceptual issues related to the role of the superior colliculus in the control of gaze. Current Opinion Neurobiology, 9, 698-707.

Stanovich, K. E., \& Pachella, R. G. (1976). The effect of stimulus probability on the speed and accuracy of naming alphanumeric stimuli. Bulletin of the Psychonomic Society, 8, 281-284.

Stein, B. E., Wallace, M. T., \& Meredith, M. A. (1995). Neural mechanisms mediating attention and orientation to multisensory cues. In M. S. Gazzaniga (Ed.), The cognitive neurosciences (pp. 683-702). Cambridge, MA: MIT Press.

Theios, J. (1975). The components of response latency in simple human information processing tasks. In P. M. A. Rabbitt \& S. Dornic (Eds.), Attention and performance $V$ (pp. 418-440). New York: Academic Press.

Wallace, M. T., Wilkinson, L. K., \& Stein, B. E. (1996). Representation and integration of multiple sensory inputs in primate superior colliculus. Journal of Neurophysiology, 76, 1246-1266.

Wurtz, R. H., \& MoHLeR, C. W. (1976). Organization of monkey superior colliculus: Enhanced visual response of superficial layer cells. Journal of Neurophysiology, 39, 745-765.

(Manuscript received March 24, 2004; revision accepted for publication February 28, 2005.) 